hep-th/0110187

\title{
Heterotic String Theory with Background Fluxes'
}

\author{
Jan Louis and Andrei Micuf \\ Fachbereich Physik, Martin-Luther-Universität Halle-Wittenberg, \\ Friedemann-Bach-Platz 6, D-06099 Halle, Germany
}

\begin{abstract}
We discuss compactifications of the heterotic string in the presence of background fluxes. Specifically we consider compactifications on $T^{6}, T^{5}, K 3 \times T^{2}$ and $K 3 \times S^{1}$ for which we derive the bosonic sector of the low energy effective action. The consistency with the corresponding gauged supergravities is demonstrated.
\end{abstract}

October 2001

\footnotetext{
${ }^{1}$ Work supported by: DFG - The German Science Foundation, GIF - the German-Israeli Foundation for Scientific Research, European RTN Program HPRN-CT-2000-00148 and the DAAD - the German Academic Exchange Service.

${ }^{2}$ email: j.louis@physik.uni-halle.de, micu@physik.uni-halle.de
} 


\section{Introduction}

Ever since its invention the heterotic string [1] has been studied as a possible candidate for a unified particle theory. In particular, its ground states with four space-time dimensions $(d=4)$ and $N=1$ supersymmetry have been of special interest due to their phenomenological attraction [2]. Such vacua arise, for example, as compactifications of the ten-dimensional heterotic string on Calabi-Yau threefolds. However, in spite of the attractive phenomenological properties there remain a few important unsolved problems such as a viable mechanism for hierarchical supersymmetry breaking and the lifting of the vacuum degeneracy.

Deformations of such vacua where non-trivial background fluxes of a $p$-form field strength are turned on have first been studied in the mid-eighties [3 5]. These fluxes arise as the "vacuum expectation values" of the field strength along appropriate cycles of the compactification manifold. The presence of the fluxes generically induce a potential for the moduli scalars which (partially) lifts the vacuum degeneracy and breaks supersymmetry [6 13]. Such vacua are special cases of so called generalized dimensional reduction introduced in [14] and further studied, for example, in refs. [15, 16].

Apart from the phenomenological issues these generalized compactifications have also been studied in connection with the various dualities which hold among seemingly different string theories [17, 18]. More specifically, ref. [19, 20] considered generalized toroidal compactifications of the heterotic string on $T^{n}$ with background fluxes turned on and showed that the perturbative T-duality $S O(n, n+16, \mathbf{Z})$ is spontaneously broken by the background fluxes. The fluxes can be arranged in appropriate representations of the T-duality group and hence the low energy effective action is invariant if the fluxes are transformed accordingly. On the other hand the non-perturbative S-duality between the heterotic string compactified on a four-torus $T^{4}$ and the type IIA string compactified on a $K 3$ manifold does not hold naively in the presence of background fluxes [21]. Similarly, the duality of the heterotic string compactified on $K 3 \times T^{2}$ and the type IIA string compactified on a Calabi-Yau threefold in the presence of fluxes has been discussed in

refs. [9, 22, 23] and in 23] it was observed that only for a very specific subset of fluxes the duality seems to be intact.

The background fluxes cannot be turned on continuously in string theory since they are quantized in units of the string scale [3]. However, in the low energy supergravity they do appear as continuous parameters and thus can be discussed as small deformations of the well known low energy effective theories derived for vanishing flux background. If no charged matter multiplets are among the massless modes the low energy supergravity turns into a gauged or massive supergravity where the fluxes play the role of the gauge charges or the mass parameters, respectively. This fact - which might not be entirely obvious a priori - thus relates various gauged supergravities to appropriate string compactifications with background fluxes. However, a complete classification is not available at present. The induced potential generically has a dilaton instability and no Minkowski or anti-de Sitter ground state exist. On the other hand non-trivial (BPS-) domain wall solutions can often be found. (See, for example, refs. [15, 16, 21]).

In this paper we focus on the derivation of the low energy effective action of generalized compactifications and in particular their relation with the known gauged supergravities. 
More specifically in section 2 we discuss the heterotic string compactified on $T^{n}$. In 2.1 we recall toroidal compactifications without background fluxes while in 2.2 we summarize the results of refs. [20] when background fluxes are turned on. The remaining sections $2.3-2.5$ then check the consistency with the known $N=4$ gauged supergravities in $d=4,5,6$. The analysis for $T^{6}$ in section 2.3 is brief since most of the work has already been done in ref. [19]. We generalize their result in that we consider a larger class of non-trivial fluxes. In section 2.4 we repeat the analysis for $T^{5}$ compactifications and show agreement with the gauged supergravity recently constructed in ref. [24]. In this section (and the associated appendix B) we give a lot more details since the result is new. As a byproduct we also derive a different form of the bosonic action and in particular a different form scalar potential for the $N=4$ gauged supergravity in $d=5$. In section 2.3 we briefly comment on the case $d=6, N=4$ where unfortunately the relevant gauged supergravity has not been constructed.[ However, our analysis suggests the form of the bosonic action of this 'yet to be constructed' gauged supergravity.

In section 3 we turn to compactifications on $K 3 \times T^{2}$. After a brief review of the ungauged heterotic theory in section 3.1 we discuss fluxes along $T^{2}$ in section 3.2. We derive the effective action and show that isometries of the vector multiplet sector are gauged. Furthermore, the consistency of the compactification with $N=2$ gauged supergravity is verified. In section 3.3 we repeat the analysis for fluxes along $K 3$ and show that now isometries in the hypermultiplet sector are gauged. Again the effective action is shown to be consistent with $N=2$ gauged supergravity. Section 3.4 considers fluxes along both $T^{2}$ and $K 3$ which corresponds to a simultaneous gauging in the vector- and hypermultiplet sector. Sections 3.2-3.4 generalize some of the aspects also discussed in ref. [23]. Finally in section 3.5 we study the closely related compactification on $K 3 \times S^{1}$ to $d=5$. We sketch the derivation of the low energy effective action and the consistency with the gauged supergravity. Section 4 contains our conclusions

Some of the technicalities are deferred to four appendices. In appendix A we record our notation and conventions. Appendix $\mathrm{B}$ contains some details of $N=4$ gauged supergravity in $d=5$ and appendix 9 contains a brief summary of gauged $N=2$ supergravity in $d=4$. In appendix $\mathrm{D}$ we derive the Killing prepotentials for the geometry obtained in section 3.3 .

\section{Toroidal compactification of the heterotic string with background fluxes}

\subsection{No background flux - the ungauged theory}

Let start by briefly recalling the standard toroidal compactification of the heterotic string without any background fluxes [18,26, 27]. The heterotic string in $d=10$ has 16 unbroken supercharges and the massless bosonic spectrum contains the metric, an antisymmetric tensor, the dilaton as well as gauge fields transforming in the adjoint representation of

\footnotetext{
${ }^{3}$ The $F(4)$ gauged supergravity coupled to matter has recently been constructed in ref. [25]. However, this does not correspond to the gauged supergravity which arises from a $T^{4}$ compactification of the heterotic string as we will argue in section 2.3.
} 
the gauge group $E_{8} \times E_{8}$ or $S O(32)$. Compactifications on an $n$-dimensional torus $T^{n}$ leave all 16 supercharges unbroken and the massless spectrum assembles in appropriate supermultiplets in $d=10-n$ space-time dimensions. The gravitational multiplet features the metric $g_{\mu \nu}, \mu, \nu=0, \ldots, d-1$, the antisymmetric tensor $B_{\mu \nu}$, the dilaton $\phi$ and $n$ graviphotons as bosonic components. All other fields reside in vector multiplets each containing a vector $A_{\mu}$ and $n$ scalar fields.

At a generic point in the field space of the scalars the non-Abelian heterotic gauge symmetry is broken to its maximal Abelian subgroup $[U(1)]^{16}$ and for most parts of the paper we are confining our attention to this Coulomb branch of the theory. In addition, there are $2 n$ Abelian gauge bosons which arise as the Kaluza-Klein gauge bosons of the metric $g_{\mu \alpha}, \alpha=1, \ldots, n$ and the antisymmetric tensor $B_{\mu \alpha}$. Thus on the Coulomb branch the total gauge group is $[U(1)]^{2 n+16}$ of which $n$ vectors reside in the gravitational multiplet while all other sit in $n+16$ vector multiplets.

Apart from the dilaton there are $n(n+16)$ moduli arising from the metric components $G_{\alpha \beta}$, the antisymmetric tensor $B_{\alpha \beta}$ and the vectors $A_{\alpha}^{a}, a=1, \ldots, 16$. Together they span the Narain moduli space [26]

$$
\mathcal{M}=\frac{S O(n, n+16)}{S O(n) \times S O(n+16)} / \Gamma_{T}
$$

where $\Gamma_{T}=S O(n, n+16, \mathbf{Z})$ is the T-duality group.

The low energy effective action is the appropriate supergravity in $d=10-n$ coupled to $16+n$ Abelian vector multiplets and is given by [27]

$$
S=\int d^{d} x \sqrt{-g} e^{-\phi}\left\{R+\left(\partial_{\mu} \phi\right)^{2}+\frac{1}{8} \partial_{\mu} M^{I J} \partial^{\mu} M_{J I}-\frac{1}{4} M_{I J} F_{\mu \nu}^{I} F^{J \mu \nu}-\frac{1}{12} H_{\mu \nu \lambda}^{2}\right\}
$$

where

$$
\begin{aligned}
F_{\mu \nu}^{I} & =\partial_{\mu} A_{\nu}^{I}-\partial_{\nu} A_{\mu}^{I}, \quad I=1, \ldots, 16+2 n, \\
H_{\mu \nu \lambda} & =\partial_{\mu} B_{\nu \lambda}-\frac{1}{2} \omega_{\mu \nu \lambda}^{\mathrm{YM}}-\frac{1}{2} \omega_{\mu \nu \lambda}^{\mathrm{G}}+\text { cyclic } .
\end{aligned}
$$

$\omega_{\mu \nu \lambda}^{\mathrm{YM}}=\eta_{I J} A_{[\mu}^{I} F_{\nu \lambda]}^{J}$ is the (Abelian) Yang-Mills Chern-Simons term while $\omega^{\mathrm{G}}$ is the gravitational Chern-Simons term which plays no role in the following. Throughout the paper the indices $I, J$ run over the total number of vector fields in the spectrum (the graviphotons plus the gauge fields in vector multiplets) and $\eta$ is the $(16+2 n)$-dimensional square matrix

$$
\eta=\left(\begin{array}{ccc}
0 & \mathbf{1}_{n} & 0 \\
\mathbf{1}_{n} & 0 & 0 \\
0 & 0 & \mathbf{1}_{16}
\end{array}\right)
$$

with signature $(n, n+16)$. $M^{I J}$ is the $S O(n, n+16)$ symmetric matrix of toroidal moduli

$$
M=\left(\begin{array}{ccc}
G^{-1} & -G^{-1} \hat{B} & -G^{-1} A \\
-\hat{B}^{T} G^{-1} & G+A^{T} A+\hat{B}^{T} G^{-1} \hat{B} & A+\hat{B}^{T} G^{-1} A \\
-A^{T} G^{-1} & A^{T}+A^{T} G^{-1} \hat{B} & \mathbf{1}_{16}+A^{T} G^{-1} A
\end{array}\right),
$$

where $\hat{B}_{\alpha \beta}=B_{\alpha \beta}+\frac{1}{2} A_{\alpha}^{a} A_{\beta}^{a}$ and we have $M_{I J}=\eta_{I K} M^{K L} \eta_{L J}=M_{I J}^{-1}$. The action (2.2) is invariant under global $S O(n, n+16)$ transformations where the vector fields transform in the vector representation $A_{\mu} \rightarrow U A_{\mu}$ while the moduli transform in the symmetric tensor representation $M \rightarrow U M U^{T}$. 


\subsection{Turning on background flux - the gauged theory}

The next step is to turn on background fluxes following ref. [20]. Let us first recall the notion of "background flux". All string theories have $(p-1)$-form gauge fields $A_{p-1}$ with a $p$-form field strength $F_{p}=d A_{p-1}$ in their massless spectrum. In a compactification the flux $m_{\Gamma_{p}}$ of the gauge field through a p-cycle $\Gamma_{p}$ is defined by the integral $m_{\Gamma_{p}}=\int_{\Gamma_{p}} F_{p}$ which can be non-vanishing. If If $A$ is globally defined $m_{\Gamma}=0$ holds by Stokes theorem. In order to have a non-vanishing flux one has to add a term to $A$ which is only locally defined. This in turn modifies the field strength $F_{p}$ by a term which is proportional to a harmonic $p$-form $\Omega_{p}$ on the compactification manifold

$$
F_{p}=d A_{p-1}+m \Omega_{p} .
$$

$m$ is constant (in space-time and on the compact manifold) and the background flux is obtained by evaluating the integral $m_{\Gamma_{p}}=\int_{\Gamma_{p}} F=m \int_{\Gamma_{p}} \Omega_{p}$. Generically the gauge invariance of $A_{p-1}$ insures the consistency of this procedure. However, whenever ChernSimons type terms are present the consistency often puts a constraint on the allowed fluxes.

Let us now summarize the results of refs. [20] where toroidal compactifications with background fluxes have been discussed. On the Coulomb branch of the ten-dimensional heterotic string there are 16 two forms $F^{a}$ and one three-form $H$. On $T^{n}$ there are the standard one-cycles while two- and three-cycles are just products of one-cycles. Consequently the non-trivial background fluxes are given by

$$
2 m_{\alpha \beta}^{a}=F_{\alpha \beta}^{a}, \quad 3 \beta_{\alpha \beta \gamma}=H_{\alpha \beta \gamma},
$$

where $m_{\alpha \beta}^{a}, \beta_{\alpha \beta \gamma}$ are constants and we follow the convention and notation of ref. [20]. In toroidal compactification additional fluxes can be turned on due to the fact that starting in $d=8$ axionic scalars appear in the spectrum as metric deformations. These are scalar fields $a$ which only appear via their field strength $d a$ in the low energy effective action. Consequently the action is invariant under a Peccei-Quinn (PQ) symmetry $a \rightarrow a+$ const. and such scalar fields can be viewed as zero-forms. Hence it is possible to include a non-vanishing background flux $\gamma_{\beta \gamma}^{\alpha}$ for their field strength (see [20] for more details). However, not all flux parameters introduced above are independent since the Bianchi identity $d H=-\frac{1}{2} F^{a} \wedge F^{a}$ imposes the constraints

$$
m_{[\alpha \beta}^{a} m_{\gamma \delta]}^{a}=3 \beta_{\epsilon[\alpha \beta} \gamma_{\gamma \delta]}^{\epsilon} .
$$

Turning on 'small' fluxes does not modify the light spectrum but does change the action. One finds [20]

$$
S=\int d^{d} x \sqrt{-g} e^{-\phi}\left\{R+\left(\partial_{\mu} \phi\right)^{2}+\frac{1}{8} \mathcal{D}_{\mu} M^{I J} \mathcal{D}^{\mu} M_{J I}-\frac{1}{4} M_{I J} F_{\mu \nu}^{I} F^{J \mu \nu}-\frac{1}{12} H_{\mu \nu \lambda}^{2}-\mathcal{V}\right\},
$$

\footnotetext{
${ }^{4}$ The flux is often called 'internal' when $\Gamma$ is a cycle on the compact manifold but not in space-time. In this paper we only consider such internal fluxes.

${ }^{5}$ Strictly speaking the Bianchi identity also includes a term $R \wedge R$ which vanishes on the torus but could contribute on a twisted torus. Following [20] we neglect this term in our analysis.
} 
where

$$
\begin{aligned}
\mathcal{D}_{\mu} M^{I J} & =\partial_{\mu} M^{I J}-f_{K L}^{I} A_{\mu}^{K} M^{L J}-f_{K L}^{J} A_{\mu}^{K} M^{I L} \\
\mathcal{V} & =\frac{1}{12} M^{I L} M^{J M} M^{K N} f_{I J K} f_{L M N}-\frac{1}{4} M^{I L} \eta^{J M} \eta^{K N} f_{I J K} f_{L M N}
\end{aligned}
$$

The $f_{K L}^{I}$ are the structure constants of a non-Abelian gauge symmetry which is a subgroup of the isometry group $S O(n, n+16)$ of the scalar manifold $\mathcal{M}$ given in (2.1). They are given in terms of the flux parameters by

$$
f_{\alpha \beta}^{a}=2 m_{\alpha \beta}^{a}, \quad f_{\alpha \beta \gamma}=-3 \beta_{\alpha \beta \gamma}, \quad f_{\beta \gamma}^{\alpha}=\gamma_{\beta \gamma}^{\alpha} .
$$

The moduli scalars are charged under the gauge group as signaled by the covariant derivatives in (2.10) and a potential $\mathcal{V}$ is induced. Accordingly also the field strength $F_{\mu \nu}^{I}$ turns into a non-Abelian field strength while $H_{\mu \nu \lambda}$ is modified by a non-Abelian Chern-Simons term [20]

$$
\begin{aligned}
F_{\mu \nu}^{I} & =\partial_{\mu} A_{\nu}^{I}-\partial_{\nu} A_{\mu}^{I}+f_{J K}^{I} A_{\mu}^{J} A_{\nu}^{K}, \\
H_{\mu \nu \lambda} & =\partial_{\mu} B_{\mu \nu}-\frac{1}{2} \omega_{\mu \nu \lambda}^{\mathrm{YM}}-\frac{1}{2} \omega_{\mu \nu \lambda}^{\mathrm{G}}+\text { cyclic }, \\
\omega_{\mu \nu \lambda}^{\mathrm{YM}} & =\eta_{I J} A_{[\mu}^{I} F_{\nu \lambda]}^{J}+\frac{1}{3} f_{I J K} A_{\mu}^{I} A_{\nu}^{J} A_{\lambda}^{K} .
\end{aligned}
$$

Apart from the local gauge symmetry the action is also manifestly invariant under the T-duality group $\Gamma_{T}=S O(n, n+16, \mathbf{Z})$ if the fluxes transform in appropriate representations of $\Gamma_{T}$. In this sense the flux parameters break the duality symmetry spontaneously. Finally, the potential has a dilaton instability due to the $e^{-\phi}$ factor in (2.9).

\subsection{The gauged supergravity of the $T^{6}$ compactification}

The purpose of this section is to show that the action (2.9) is a special case of the most general action of gauged $d=4, N=4$ supergravity as constructed in ref. [28. This generalizes the analysis of ref. [19] where a subset of fluxes was considered. More specifically ref. [19] started from the standard heterotic string compactified on $T^{5}$ without any background fluxes and performed a further $S^{1}$ reduction with background fluxes turned on along this $S^{1}$. It was shown that the resulting bosonic action is a special case of the gauged supergravity of ref. [28]. Here we generalize this analysis in that we show that also for arbitrary flux parameters as considered in [20] the action (2.9) is a special case of the bosonic action constructed in [28]. In doing so we closely follow the procedure of ref. "19..

In $d=4, N=4$ the vector multiplet contains 6 scalar fields $A_{\alpha}, \alpha=1, \ldots, 6$. In $T^{6}$ compactifications of the heterotic string there are $16+6=22$ such Abelian vector

\footnotetext{
${ }^{6}$ The indices on $f$ are raised and lowered with $\eta$. Strictly speaking, they should be raised and lowered with the Cartan metric which is not possible here since the gauge group is generically not semi-simple. However, due to the fact that this group is embedded in the isometry group $S O(n, n+16)$, we can raise and lower the indices with $\eta$ which is the metric on the coset $\mathcal{M}$. (See also the discussion in [20].)
} 
multiplets containing $6 \cdot 22$ scalar fields which span the coset $S O(6,22) / S O(6) \times S O(22)$. These scalar fields are conveniently assembled in a $28 \times 6$ matrix $Z_{\alpha}^{I}$ which satisfies the $S O(6,22)$ invariant constraint

$$
Z_{\alpha}^{I} \eta_{I J} Z_{\beta}^{J}=-\delta_{\alpha \beta}
$$

We do not recall the standard $N=4$ action in terms of the field variables $Z_{\alpha}^{I}$ here and instead refer the reader to the literature [19, 28, 29]. In order to compare this $N=4$ action with the action displayed in (2.9) one has to go a different set of coordinates defined by $[19$

$$
M^{I J}=\eta^{I J}+2 Z_{\alpha}^{I} Z_{\beta}^{J} \delta^{\alpha \beta}
$$

which are elements of $S O(6,22)$ in that they satisfy $M \eta M=\eta$. In the string frame the $N=4$ supergravity action - now expressed in terms of the $M$ variables - is indeed given by eq. (2.9) as was shown in ref. [19]. However, the most general possible $N=4$ potential reads 19, 28

$$
\mathcal{V}=\frac{1}{12}\left(2 \eta^{I L} \eta^{J M} \eta^{K N}-3 \eta^{I L} \eta^{J M} M^{K N}+M^{I L} M^{J M} M^{K N}\right) f_{I J K} f_{L M N}
$$

Exactly as in the case of toroidal compactifications the $f_{I J K}$ are the structure constants of the non-Abelian gauge symmetry. The difference being that in toroidal compactifications the $f_{I J K}$ are in turn determined by the background fluxes according to eq. (2.11). By inspection we see that the potential obtained in eq. (2.10) corresponds to the last two terms of the potential given in (2.15). What is left to show is that the first term in (2.15) vanishes for the specific fluxes which arise in $T^{6}$ compactifications and are given in eq. (2.11). This first term in (2.15) is nothing but the contraction of the Killing form $K_{I J}=f_{I K}^{L} f_{L J}^{K}$ with $\eta$. Due to the special form of the structure constants (2.11), the only non-zero components of the Killing form are

$$
K_{I J}=8 \gamma_{\alpha \delta}^{\gamma} \gamma_{\gamma \beta}^{\delta} \delta_{I}^{\alpha} \delta_{J}^{\beta}
$$

Using (2.4) it follows immediately that $\eta^{I J} K_{J I}=0$ and hence

$$
\eta^{I L} \eta^{J M} \eta^{K N} f_{I J K} f_{L M N}=0
$$

To summarize, we just showed that in $d=4$ the action obtained from generalized toroidal $T^{6}$ compactification of the heterotic string with non-trivial background fluxes as obtained in refs. [19, 20] is a special case of the most general action of gauged $N=4$ supergravity constructed in refs. [28]. 8

\subsection{The gauged supergravity of the $T^{5}$ compactification}

The next step is to redo the previous analysis for $T^{5}$ compactifications and show the consistency with the gauged supergravity recently constructed in ref. [24]. In $d=5$ the

\footnotetext{
${ }^{7}$ The reason is that $T^{6}$ compactifications are already discussed in ref. [19] and here we only slightly generalize their analysis. Furthermore, in the next section when we discuss $T^{5}$ compactifications we give a lot more details and they strongly resemble the $T^{6}$ situation.

${ }^{8}$ It would be interesting to see if there are string backgrounds where $\eta^{I J} K_{I J} \neq 0$ holds such that the first term in (2.15) does not vanish anymore.
} 
gravitational multiplet contains apart from the metric and the dilaton 6 vector fields which transform in the $\mathbf{5}+\mathbf{1}$ representation of the R-symmetry group $U S p(4) \sim S O(5)$. The singlet plays the role of a 'spectator' gauge field and will later on be dualized to the antisymmetric tensor $B_{\mu \nu}$ of the heterotic string. In addition the spectrum features 21 vector multiplets each containing 5 scalar fields. So apart from the dilaton there are $5 \cdot 21$ scalars parameterizing the coset $S O(5,21) / S O(5) \times S O(21)$. In ref. [24 the scalar fields are written as $S O(5,21)$ matrices $\left(L_{I}^{[\Sigma \Lambda]}, L_{I}{ }^{i}\right)$ satisfying the constraints

$$
\begin{aligned}
L_{I i} L_{J}^{i}-L_{I[\Sigma \Lambda]} L_{J}^{[\Sigma \Lambda]} & =\eta_{I J}, \\
-L_{I}^{\Sigma \Lambda} L_{J \Delta \Omega} \eta^{I J} & =\delta_{\Delta}^{[\Sigma} \delta_{\Omega}^{\Lambda]}-\frac{1}{4} \Omega^{\Sigma \Lambda} \Omega_{\Delta \Omega}, \\
L_{I i} L^{I j} & =\delta_{i}^{j},
\end{aligned}
$$

where $\Sigma, \Lambda$ are $U S p(4)$ indices $\Sigma, \Lambda=1, \ldots, 4$ and $\Omega_{\Delta \Omega}$ is the invariant symplectic twoform of $U S p(4)$. The $L_{I[\Sigma \Lambda]}$ transform in the six-dimensional representation of $U S p(4)$ while the $L_{I i}$ reside in the vector representation of $S O(21)$, i.e. $i=1, \ldots, 21$ (for more details see appendix B).

In ref. 24] it was shown that different subgroups $K$ of $S O(5,21)$ can be gauged and if $K$ does not contain an Abelian factor - as is the case in $T^{5}$ compactifications of the heterotic string - the bosonic part of the gauged supergravity action in the Einstein frame is given by 24

$$
\begin{aligned}
S= & \int d^{5} x \sqrt{-g}\left[\frac{1}{2} R-\frac{1}{2}\left(\partial_{\mu} \phi\right)^{2}-\frac{1}{4} e^{\frac{2 \phi}{\sqrt{3}}} M_{I J} F_{\mu \nu}^{I} F^{\mu \nu J}-\frac{1}{4} e^{-\frac{4 \phi}{\sqrt{3}}} G_{\mu \nu} G^{\mu \nu}\right. \\
& \left.-\frac{1}{2} P_{\mu}^{i[\Sigma \Lambda]} P_{i[\Sigma \Lambda]}^{\mu}-e^{\frac{-2 \phi}{\sqrt{3}}} \mathcal{V}\right]+\frac{\sqrt{2}}{8} \int d^{5} x \eta_{I J} F_{\mu \nu}^{I} F_{\rho \sigma}^{J} a_{\lambda} \epsilon^{\mu \nu \rho \sigma \lambda},
\end{aligned}
$$

where the 'spectator' gauge field is denoted by $a_{\mu}$ and its field strength by $G_{\mu \nu}$. The kinetic terms for the scalars involve

$$
P_{\mu}^{i[\Sigma \Lambda]} \equiv L_{I}^{i} \mathcal{D}_{\mu} L^{I[\Sigma \Lambda]}
$$

where $\mathcal{D}_{\mu}$ is the covariant derivative with respect to the gauge group and it can be found explicitly in ref. [24]. The gauge couplings are given by

$$
M_{I J}=L_{I[\Sigma \Lambda]} L_{J}^{[\Sigma \Lambda]}+L_{I i} L_{J}^{i}
$$

while the scalar potential reads

$$
\mathcal{V}=2\left(L^{J i} L_{i}^{M} L^{K}{ }_{\Sigma}{ }^{\Omega} L_{I \Omega \Lambda} L^{N \Sigma \Delta} L_{L \Delta}{ }^{\Lambda}-\frac{4}{9} L^{J}{ }_{\Sigma \Omega} L_{I}{ }^{\Omega \Delta} L^{K}{ }_{\Delta \Lambda} L^{M \Sigma}{ }_{\Xi} L_{L}{ }^{\Xi \Gamma} L^{N}{ }_{\Gamma}^{\Lambda}\right) f_{J K}^{I} f_{M N}^{L} .
$$

So far we just recalled the results of ref. [24]. Now we have to show that the action (2.9) for $d=5$ is a special case of (2.19). In particular this involves the identification of the potential, the kinetic terms for the moduli and the dualization of the spectator gauge field $a_{\lambda}$ in terms of an antisymmetric tensor $B_{\mu \nu}$. First let us note that the matrix

\footnotetext{
${ }^{9}$ Anticipating the result we denote the gauge couplings by $M_{I J}$ instead of $a_{I J}$ used in [24.
} 
$L_{I}{ }^{i}$ is the only bosonic quantity which carries an $S O(21)$ vector index $i$. Hence it always appears quadratically in the action and thus can be eliminated using (2.18). Th We are then left with the matrices $L_{\Sigma \Lambda}^{I}$ describing the scalar manifold. As before it is convenient to define a new set of matrices $Z_{\alpha}^{I}$ by

$$
Z_{\alpha}^{I}=\frac{1}{2}\left(\Gamma_{\alpha}\right)_{\Lambda}{ }^{\Sigma} L_{\Sigma}^{I}{ }^{\Lambda}
$$

where the $\Gamma^{\alpha}$ are Euclidean $4 \times 4 \Gamma$-matrices. The conventions are summarized in appendix $\mathrm{B}$ where we also show the identities

$$
Z_{\alpha}^{I} Z_{\beta}^{J} \eta_{I J}=-\delta_{\alpha \beta}, \quad L_{I}{ }^{\Sigma \Lambda} L_{J \Sigma \Lambda}=Z_{\alpha I} Z_{\beta J} \delta^{\alpha \beta} \equiv Z_{I J}
$$

Inserting (2.24) into (2.21) and using (2.18) we arrive at

$$
M_{I J}=\eta_{I J}+2 Z_{I J}
$$

which is the five-dimensional analog of eq. (2.14). With the help of (2.25) we are able to rewrite the kinetic term for the scalars in terms of the matrix $M_{I J}$. Starting from its definition (2.20) and using (2.18), (2.24), (B.4) we obtain

$$
\begin{aligned}
\frac{1}{2} P_{\mu}^{i \Sigma \Lambda} P_{i \Sigma \Lambda}^{\mu} & =\frac{1}{2} L_{I}^{i} \mathcal{D}_{\mu} L^{I \Sigma \Lambda} L_{J i} \mathcal{D}^{\mu} L_{\Sigma \Lambda}^{J}=\frac{1}{2}\left(\eta_{I J}+Z_{I J}\right) \mathcal{D}_{\mu} Z_{\alpha}^{I} \mathcal{D}^{\mu} Z_{\beta}^{J} \delta^{\alpha \beta} \\
& =-\frac{1}{2} \eta_{I J} \eta_{K L} Z_{\alpha}^{K} Z_{\beta}^{L} \mathcal{D}_{\mu} Z_{\alpha}^{I} \mathcal{D}^{\mu} Z_{\beta}^{J}+\frac{1}{2} \eta_{I K} \eta_{J L} Z_{\alpha}^{K} Z_{\alpha}^{L} \mathcal{D}_{\mu} Z_{\beta}^{I} \mathcal{D}^{\mu} Z_{\beta}^{J} .
\end{aligned}
$$

Taking the covariant derivative of (2.24) one also derives

$$
Z_{\beta}^{J} \mathcal{D}_{\mu} Z_{\alpha}^{I} \eta_{I J}=-Z_{\alpha}^{I} \mathcal{D}_{\mu} Z_{\beta}^{J} \eta_{I J}
$$

which when inserted into (2.26) results in

$$
\frac{1}{2} P_{\mu}^{i \Sigma \Lambda} P_{i \Sigma \Lambda}^{\mu}=-\frac{1}{4} \eta_{I K} \eta_{J L} \mathcal{D}_{\mu} Z^{I J} \mathcal{D}^{\mu} Z^{K L}=-\frac{1}{16} \mathcal{D}_{\mu} M^{I J} \mathcal{D}^{\mu} M_{J I}
$$

(The last equality used (2.25) and again the fact that $\eta_{I J}$ is covariantly constant.)

Next we also rewrite the potential in terms of $M_{I J}$. Starting from (2.22) using (2.18) and (B.5) we obtain

$$
\begin{aligned}
\mathcal{V}=-\frac{1}{8}[ & \left(Z^{I L}+\eta^{I L}\right) Z_{\alpha}^{J} Z_{\beta}^{K} Z_{\gamma}^{M} Z_{\delta}^{N} \operatorname{Tr}\left(\Gamma^{\alpha} \Gamma^{\beta} \Gamma^{\gamma} \Gamma^{\delta}\right) \\
& \left.-\frac{1}{9} Z_{\alpha}^{I} Z_{\beta}^{J} Z_{\gamma}^{K} Z_{\delta}^{L} Z_{\epsilon}^{M} Z_{\phi}^{N} \operatorname{Tr}\left(\Gamma^{\alpha} \Gamma^{\beta} \Gamma^{\gamma} \Gamma^{\delta} \Gamma^{\epsilon} \Gamma^{\phi}\right)\right] f_{I J K} f_{L M N} .
\end{aligned}
$$

The traces can be computed using $(\overline{B .3})$ and taking into account the antisymmetry of the structure constants the form of the potential is found to be

$$
\begin{aligned}
\mathcal{V} & =Z^{J M} Z^{K N}\left(\eta^{I L}+\frac{2}{3} Z^{I L}\right) f_{I J K} f_{L M N} \\
& =\frac{1}{12}\left(2 \eta^{I L} \eta^{J M} \eta^{K N}-3 \eta^{I L} \eta^{J M} M^{K N}+M^{I L} M^{J M} M^{K N}\right) f_{I J K} f_{L M N},
\end{aligned}
$$

\footnotetext{
${ }^{10}$ For the fermionic part of the Lagrangian, the discussion is more involved and we will not get into details here.
} 
where the last equality used again (2.25). We see that the potential of the $d=5$ action can be rewritten exactly in the same form as in the $d=4$ action. This result is a byproduct of our analysis. By the exact same argument as in $d=4$ the first term in the potential vanishes for the fluxes turned on in $T^{5}$ compactifications (2.11). Inserting (2.28), (2.30) into 2.19) and performing a Weyl rescaling of the metric we arrive at

$$
\begin{aligned}
S= & \frac{1}{2} \int d^{5} x \sqrt{-g} e^{-\phi}\left[R+\left(\partial_{\mu} \phi\right)^{2}+\frac{1}{8} \mathcal{D}_{\mu} M^{I J} \mathcal{D}^{\mu} M_{J I}-\mathcal{V}\right. \\
& \left.-\frac{1}{4} M_{I J} F_{\mu \nu}^{I} F^{J \mu \nu}-\frac{1}{4} e^{2 \phi} G_{\mu \nu} G^{\mu \nu}\right]+\frac{1}{8} \int d^{5} x \eta_{I J} F_{\mu \nu}^{I} F_{\rho \sigma}^{J} a_{\lambda} \epsilon^{\mu \nu \rho \sigma \lambda} .
\end{aligned}
$$

The final step is to dualize the spectator gauge field $a_{\lambda}$ to an antisymmetric tensor. The last term in (2.31) will give upon dualization the right form of the Chern-Simons correction to $H$ which on the string theory side is dictated by anomaly cancellation. The details can be found in appendix B with the final result being the action of eq. (2.9).

\subsection{Comments on $T^{4}$ compactification}

The same analysis as in the previous two sections can be carried out for $T^{4}$. Unfortunately, the most general $N=2$ (16 supercharges) gauged supergravity in six dimensions has not been constructed yet. In particular, the supergravity recently derived in ref. 25 does not include the gauged supergravity which is obtained by compactification with fluxes. The reason is that the group which is gauged in 25] is a direct product of $S U(2) \subset S O(4) \subset S O(4, m)$ and a semi-simple group $G \subset S O(m) \subset S O(4, m)$, while the group which becomes gauged due to the presence of fluxes in the compactification on $T^{4}$ can not be put in such a form. However, one can naturally generalize the result of [25] (or at least the potential) to accommodate bigger groups $G^{\prime} \subset S O(4, m)$. $\square$ This procedure leads to a potential of the form (2.30) which, as it was argued in the previous sections, is in agreement with the potential which comes from compactification.

\section{$3 \quad K 3 \times T^{2}$ compactification with fluxes}

\subsection{No background flux - the ungauged theory}

Let us turn to compactifications of the heterotic string on $K 3 \times T^{2}$ and first briefly review the ungauged theory 30 34. This class of heterotic string vacua has four flat Minkowski dimensions and leaves 8 supercharges unbroken. Thus the low energy effective theory is an $N=2$ supergravity coupled to $n_{V}$ vector- and $n_{H}$ hypermultiplets. ${ }^{12}$ The precise

\footnotetext{
${ }^{11}$ The precise way to do this is more involved and to give more details here would mean to introduce the whole notation used in 25]. The main idea is to put the groups $S U(2)$ and $G$ on the same footing and use generic structure constants which belong to a group $G^{\prime} \supset S U(2) \times G$. We are grateful to Marco Zagermann for clarifying this point.

${ }^{12}$ Strictly speaking the heterotic dilaton resides in a vector-tensor multiplet 31] but this subtlety will play no role here. A short review about $N=2$ supergravity is assembled in appendix C.
} 
number of vector- and hypermultiplets depends on the details compactifications and in particular on the specific solution of the constraint

$$
\int_{K 3} d H=\int_{K 3}(\operatorname{tr} R \wedge R-\operatorname{tr} F \wedge F)=24-\int_{K 3} \operatorname{tr} F \wedge F=0 .
$$

(3.1) implies that the gauge bundle on $K 3$ is necessarily non-trivial with instanton numbers which have to add up to 24 . This in turn breaks the heterotic gauge group and introduces a set of bundle moduli fields which parameterize the embedding of the instantons in the gauge bundle. Furthermore, depending on the embedding of the instantons charged hypermultiplets appear among the massless modes. In the following we do not need to discuss the generic situation since we will only consider fluxes for (Abelian) gauge fields under which no hypermultiplet is charged. Hence in this section we discuss a spectrum of $n_{V}$ Abelian vector- and $n_{H}$ gauge neutral hypermultiplets.

The low energy effective action can be derived by performing the standard KaluzaKlein reduction of the ten-dimensional heterotic theory. The final action is known for some time [30 33] but for later use we present some details in the following. After an appropriate Weyl rescaling and the dualization of the antisymmetric tensor $B_{\mu \nu}$ to an axionic scalar $a$, one finds

$$
\begin{gathered}
S=2 \int d^{4} x \sqrt{-g}\left[\frac{1}{2} R+\frac{1}{4} I_{I J} F_{\mu \nu}^{I} F^{J \mu \nu}+\frac{1}{8} R_{I J} F_{\mu \nu}^{I} F_{\rho \lambda}^{J} \epsilon^{\mu \nu \rho \lambda}\right. \\
\left.+\frac{\partial_{\mu} s \partial^{\mu} \bar{s}}{(s-\bar{s})^{2}}+\frac{1}{16} \partial_{\mu} M^{I J} \partial^{\mu} M_{J I}-h_{u v} \partial_{\mu} q^{u} \partial^{\mu} q^{v}\right]
\end{gathered}
$$

where the $q^{u}, u=1, \ldots, 4 n_{h}$ denote the scalars in the hypermultiplets and $h_{u v}$ is their quaternionic metric. Due to the presence of the bundle moduli which reside in hypermultiplets this metric is largely unknown. $N=2$ supergravity constrains the metric to be quaternionic and furthermore in $K 3$ compactifications the moduli space of the hypermultiplets $\mathcal{M}_{H}$ has a submanifold spanned by the moduli of the $K 3$ surface which is given by 35

$$
\mathcal{M}_{H} \supset \mathcal{M}_{K 3}=\frac{S O(4,20)}{S O(4) \times S O(20)} .
$$

The variable $s$ in (3.2) denotes the heterotic dilaton which is defined as

$$
s=\frac{a}{2}-\frac{i}{2} e^{-\phi}
$$

and the gauge couplings are found to be

$$
I_{I J}=\frac{(s-\bar{s})}{2 \imath} M_{I J}, \quad R_{I J}=-\frac{s+\bar{s}}{2} \eta_{I J},
$$

where the matrix $M_{I J}$ is defined in (2.5). The dilaton $s$ together with the toroidal moduli of $M_{I J}$ form the moduli of the vector multiplets and $N=2$ supergravity requires that they span a special Kähler manifold $\mathcal{M}_{V}$ (for more details see appendix C). For the heterotic string compactified on $K 3 \times T^{2}$ this known to be 3034

$$
\mathcal{M}_{V}=\frac{S U(1,1)}{U(1)} \otimes \frac{S O\left(2, n_{V}-1\right)}{S O(2) \times S O\left(n_{V}-1\right)}
$$


where the $\frac{S U(1,1)}{U(1)}$ factor is spanned by the heterotic dilaton multiplet $s$. Indeed, one can find appropriate complex Kähler coordinates by the transformation 22]

$$
\begin{aligned}
A_{1}^{a} & =\sqrt{2} \frac{n^{a}-\bar{n}^{a}}{u-\bar{u}}, \quad A_{2}^{a}=\sqrt{2} \frac{\bar{u} n^{a}-u \bar{n}^{a}}{u-\bar{u}}, \\
B_{12} & =\frac{1}{2}\left[(t+\bar{t})-\frac{(n+\bar{n})^{a}(n-\bar{n})^{a}}{u-\bar{u}}\right] \\
\sqrt{G} & =-\frac{\imath}{2}\left[(t-\bar{t})-\frac{(n-\bar{n})^{a}(n-\bar{n})^{a}}{u-\bar{u}}\right], \\
G_{11} & =\frac{2 \imath}{u-\bar{u}} \sqrt{G}, \quad G_{12}=\imath \frac{u+\bar{u}}{u-\bar{u}} \sqrt{G} .
\end{aligned}
$$

In this new field basis the scalars of the vector multiplets are collectively denoted by $z^{i}=\left(s, t, u, n^{a}\right), a=4, \ldots, n_{V}$. Inserting (3.7) into (3.2) using (2.5) the action is found to be

$$
S=2 \int d^{4} x \sqrt{-g}\left[\frac{1}{2} R+\frac{1}{4} I_{I J} F_{\mu \nu}^{I} F^{J \mu \nu}+\frac{1}{8} R_{I J} F_{\mu \nu}^{I} F_{\rho \lambda}^{J} \epsilon^{\mu \nu \rho \lambda}-g_{i \bar{\jmath}} \partial_{\mu} z^{i} \partial^{\mu} \bar{z}^{\bar{\jmath}}-h_{u v} \partial_{\mu} q^{u} \partial^{\mu} q^{v}\right],
$$

where the metric $g_{i \bar{\jmath}}$ is Kähler, i.e. $g_{i \bar{\jmath}}=\partial_{i} \partial_{\bar{\jmath}} K$ with

$$
K=-\ln \imath(\bar{s}-s)-\ln \frac{1}{4}\left[(t-\bar{t})(u-\bar{u})-(n-\bar{n})^{a}(n-\bar{n})^{a}\right] .
$$

This is the Kähler potential of the coset (3.6).

In the following we choose to relabel the indices in order to be consistent with the standard $N=2$ conventions. More specifically, the index $I$ which runs over all $n_{V}+1$ vector fields of the theory starts at $I=0$, i.e. $I=0, \ldots, n_{V}$ where $I=0$ denotes the graviphoton. Furthermore, the matrix $\eta$ defined in eq. (2.4) is relabeled such that $\eta_{01}=\eta_{10}=\eta_{23}=\eta_{32}=\eta_{a a}=1$ while all other matrix elements vanish. The matrix $M_{I J}$ is relabeled accordingly and conventionally written as

$$
M_{I J}=\eta_{I J}-2 \frac{\left(X_{I} \bar{X}_{J}+\bar{X}_{I} X_{J}\right)}{X^{I} \eta_{I J} \bar{X}^{J}},
$$

where the coordinates $X^{I}, I=0, \ldots, n_{V}$ are related to the toroidal moduli via

$$
X^{0}=\frac{1}{2}, \quad X^{1}=\frac{1}{2}\left(u t-n^{a} n^{a}\right), \quad X^{2}=-\frac{1}{2} u, \quad X^{3}=\frac{1}{2} t, \quad X^{a}=\frac{1}{\sqrt{2}} n^{a},
$$

and we defined $X_{I} \equiv \eta_{I J} X^{J}$. In these coordinates the Kähler potential (3.9) reads

$$
K=-\ln \imath(\bar{s}-s)-\ln X^{I} \eta_{I J} \bar{X}^{J} .
$$

Note that the coordinates $X^{I}$ are not all independent but satisfy the constraint

$$
X^{I} \eta_{I J} X^{J}=0
$$

This is related to the fact that in the heterotic string one naturally obtains a basis of field variables which differ from the standard $N=2$ convention discussed in appendix $\mathrm{C}$ by 
a symplectic rotation [30 34. In the standard field variables the coset (3.6) is described by a holomorphic prepotential

$$
F=\frac{X^{1}\left(X^{2} X^{3}-X^{a} X^{a}\right)}{X^{0}}
$$

By performing the symplectic rotation $X^{1} \rightarrow-F_{1}, F_{1} \rightarrow X^{1}$, one obtains the field basis used in (3.11) where the constraint (3.13) holds and a holomorphic prepotential does not exist 30 34.

Now we are prepared to discuss the theory with background fluxes turned on. We perform the analysis in two steps. First we compactify the ten-dimensional heterotic theory on $T^{2}$ with all possible fluxes turned on and then do a further compactification on $K 3$ where all fluxes along the $K 3$ vanish. In a second step we switch off the fluxes along $T^{2}$ but now consider all possible fluxes along the $K 3$. Finally we discuss the combined situation. Certain aspects of this analysis have also been done in ref. [23].

\subsection{Flux along $T^{2}$}

For the $T^{2}$ compactification with fluxes we can straightforwardly use the the results of [20] which we reviewed in section 2.2. For a two-torus the formuli considerably simplify. First of all no $H$-fluxes can be turned on since that needs at least three internal indices, i.e. a $T^{3}$. Furthermore in the gravitational sector an internal axion only starts to appear in $d=8$, as we discussed in section 2.2. This means that non-zero fluxes can only be turned on for the field strength of the gauge fields which are already present in $d=10$. Consequently, the only non-vanishing structure constants which we obtain from eq. (2.11) on $T^{2}$ are $f_{12}^{a}=2 m^{a}$. In the relabeled field basis introduced in the previous section the non-vanishing structure constants become

$$
f_{30}^{a}=2 m^{a} .
$$

Inserted into (2.10) the potential dramatically simplifies and reads

$$
\mathcal{V}=2 \operatorname{det}\left(G^{-1}\right) m^{a} m^{a} .
$$

The compactification of the eight-dimensional version of the action (2.9) on $K 3$ proceeds exactly as in the ungauged case and results in

$$
\begin{gathered}
S=2 \int d^{4} x \sqrt{-g}\left[\frac{1}{2} R+\frac{1}{4} I_{I J} F_{\mu \nu}^{I} F^{J \mu \nu}+\frac{1}{8} R_{I J} F_{\mu \nu}^{I} F_{\rho \lambda}^{J} \epsilon^{\mu \nu \rho \lambda}\right. \\
\left.-g_{i \bar{\jmath}} \mathcal{D}_{\mu} z^{i} \mathcal{D}^{\mu} \bar{z}^{\bar{\jmath}}-h_{u v} \partial_{\mu} q^{u} \partial^{\mu} q^{v}-\mathcal{V}_{\mathrm{E}}\right]
\end{gathered}
$$

where the covariant derivatives are given by ${ }^{\text {th }}$

$$
\begin{aligned}
\mathcal{D}_{\mu} u & =\partial_{\mu} u, \quad \mathcal{D}_{\mu} s=\partial_{\mu} s, \\
\mathcal{D}_{\mu} t & =\partial_{\mu} t-2 \sqrt{2} A_{\mu}^{3} m^{a} n^{a}+2 m^{a} A_{\mu}^{a}, \\
\mathcal{D}_{\mu} n^{a} & =\partial_{\mu} n^{a}-\sqrt{2}\left(A_{\mu}^{3} u+A_{\mu}^{0}\right) m^{a} .
\end{aligned}
$$

\footnotetext{
${ }^{13}$ Notice that the indices are now raised and lowered with the 'new' $\eta$-matrix and hence we have $f_{30}^{a}=f_{0 a}^{2}=f_{a 3}^{1}$.

${ }^{14}$ These covariant derivatives are nothing but the covariant derivatives of eq. (2.10) expressed in the coordinates (3.7) with the specific choice of structure constants given in (3.15).
} 
The potential $\mathcal{V}_{\mathrm{E}}$ is obtained from (3.16) by an appropriate Weyl rescaling and inserting the coordinate transformations (3.7) one finds

$$
\mathcal{V}_{\mathrm{E}} \equiv \frac{-\imath \mathcal{V}}{2(s-\bar{s})}=\frac{4 \imath(u-\bar{u})^{2} m^{a} m^{a}}{(s-\bar{s})\left[(n-\bar{n})^{2}-(u-\bar{u})(t-\bar{t})\right]^{2}}
$$

We see that compared to the ungauged action (3.2) the fluxes induce a potential $\mathcal{V}_{\mathrm{E}}$ and turn some of the derivatives of the scalars in the vector multiplets into covariant derivatives. Thus a subgroup of the isometry group of the vector multiplets is gauged by the presence of the fluxes $m^{a}$.

We can check the consistency of the low energy effective theory with the standard $N=2$ gauged supergravity which we briefly summarize in appendix C. The generic form of the covariant derivatives of the scalars is given by

$$
\mathcal{D}_{\mu} z^{i}=\partial_{\mu} z^{i}-k_{I}^{i} A_{\mu}^{I}
$$

where the $k_{I}^{i}$ are the Killing vectors of the isometries $\delta z^{i}=\Lambda^{I} k_{I}^{i}$. Comparing with (3.18) we can read off the non-zero Killing vectors

$$
k_{3}^{t}=2 \sqrt{2} m^{a} n^{a}, \quad k_{a}^{t}=-2 m^{a}, \quad k_{0}^{n^{a}}=\sqrt{2} m^{a}, \quad k_{3}^{n^{a}}=\sqrt{2} m^{a} u .
$$

In the case that only isometries in the vector multiplet sector are gauged the general $N=2$ potential given in (C.12) simplifies and reads

$$
\mathcal{V}_{\mathrm{E}}=e^{K} g_{i \bar{\jmath}} k_{I}^{i} k_{J}^{\bar{\jmath}} \bar{X}^{I} X^{J}
$$

Inserting (3.21) into (3.22) and using the definitions (3.11) one verifies the consistency with the potential given in (3.19).

\subsection{Fluxes on $K 3$}

Let us now discuss the case where non-trivial background fluxes along the $K 3$ but not along $T^{2}$ are turned on. On $K 3$ there are 22 harmonic two-forms $\Omega_{A}$ and one harmonic four-form $\hat{\Omega}_{(4)}$ but no three-form. Hence, the possible fluxes can only arise from the gauge field strength $F$ while the three-form $H$ cannot contribute. More precisely, we expand the (Abelian) gauge fields with vanishing instanton number or in other words the gauge fields which are not involved in the solution of the constraint (3.1) according to

$$
F^{I}=F^{I}(x)+m^{I A} \Omega_{A}, \quad A=1, \ldots, 22,
$$

where $F^{I}(x)$ is the field strength in the four-dimensional space-time and $\Omega_{A}$ are the 22 harmonic two-forms on $K 3$. Furthermore, we insist that no hypermultiplet is charged under the gauge fields for which $m^{I A} \neq 0$. 四

Since we are turning on the fluxes perturbatively the constraint (3.1) is modified. However, we do not change the instanton configuration and continue to choose part of

\footnotetext{
${ }^{15}$ The case where hypermultiplets are charged with respect to gauge fields with fluxes is discussed in refs. [7, 9].
} 
the gauge group with non-trivial instanton number, i.e. $\int_{K 3} t r F^{2}=24$. In order to satisfy (3.1) the additional fluxes $m^{A I}$ then have to obey

$$
0=\int_{K 3} \eta_{I J} F^{I} \wedge F^{J}=m^{A I} m^{B J} \rho_{A B} \eta_{I J}
$$

where $\rho$ denotes the intersection matrix on the second cohomology $H^{2}(K 3)$

$$
\rho_{A B}=\int_{K 3} \Omega_{A} \wedge \Omega_{B}
$$

$\rho$ has signature $(3,19)$ and is left invariant by $S O(3,19)$. Note that the condition (3.24) is the equivalent of (2.8) for toroidal compactifications.

Due to the Chern-Simons couplings the action cannot be written only in terms of $F$ and thus the consistency of the reduction is not entirely obvious. However, by dualizing the antisymmetric tensor $B$ in eight dimension to a four-form $C_{4}$ 23 the kinetic term $\int e^{-\phi} H_{\mu \nu \rho}^{2}$ turns into

$$
S_{C}=\int e^{\phi} G_{5}^{2}-\int C_{4} \wedge F^{I} \wedge F^{J} \eta_{I J}
$$

where $G_{5}=d C_{4}$. In this dual basis the Kaluza-Klein reduction can be performed with $F^{I}$ given in (3.23) and the four-form $C_{4}$ expanded according to

$$
C_{4}=c^{A}(x) \wedge \Omega_{A}+a(x) \hat{\Omega}_{(4)} .
$$

The $c^{A}$ are 22 two-forms in space-time while $\hat{\Omega}_{(4)}$ is the volume form on $K 3$ and $a(x)$ the associated scalar field. tensors $c^{A}$ into axionic scalar fields $b^{A}$. $(a(x)$ is the axion which arises from dualizing $B_{\mu \nu}$ in $d=4$.) The final action in the $d=4$ Einstein frame reads

$$
\begin{aligned}
S= & 2 \int d^{4} x \sqrt{-g}\left[\frac{1}{2} R+\frac{1}{8} I_{I J} F_{\mu \nu}^{I} F^{J \mu \nu}+\frac{1}{16} R_{I J} F_{\mu \nu}^{I} F_{\rho \lambda}^{J} \epsilon^{\mu \nu \rho \lambda}\right. \\
& \left.-g_{i j} \partial_{\mu} z^{i} \partial^{\mu} \bar{z}^{\bar{j}}-h_{u v} \mathcal{D}_{\mu} q^{u} \mathcal{D}^{\mu} q^{v}-\mathcal{V}_{\mathrm{E}}\right]
\end{aligned}
$$

where among the hypermultiplet scalars $q^{u}$ only the 22 axions $b^{A}$ are charged and their covariant derivatives are given by

$$
\mathcal{D}_{\mu} b^{A}=\partial_{\mu} b^{A}-m_{I}^{A} A_{\mu}^{I} .
$$

The potential is found to $\mathrm{be}$

$$
\mathcal{V}_{\mathrm{E}}=\frac{2}{(s-\bar{s})^{2}} h_{A B} m^{A I} m^{B J} I_{I J}
$$

where $h_{A B}$ is the restriction of the quaternionic metric $h_{u v}$ to the space spanned by $b^{A}$ and is given by:

$$
h_{A B}=\frac{1}{4 v} N_{A B}, \quad N_{A B}(q)=\int_{K 3} \Omega_{A} \wedge^{*} \Omega_{B} .
$$

\footnotetext{
${ }^{16}$ The space-time part of $C_{4}$ does not contribute since it does not have a kinetic term and a possible contribution to the potential vanishes due to 3.24 .

${ }^{17}$ For a subset of fluxes and at special points in the $K 3$ moduli space this potential has also been obtained in ref. [23.
} 
Here $v$ is the volume modulus of $K 3$ while $N_{A B}$ depends only on the remaining 57 moduli of $K 3$ and not on the 22 axions $b^{A}$. From (3.29) we see that contrary to the previous case (fluxes along $T^{2}$ ) the non-trivial fluxes along $K 3$ gauge isometries in the hypermultiplet moduli space $\mathcal{M}_{H}$.

As before the final step is to check the consistency with the standard gauged supergravity. Inserting (3.5) into (C.12) using (3.10) and $k_{I}^{i}=0$ one finds that the gauging in the hypermultiplet sector induces the potential

$$
\mathcal{V}_{\mathrm{E}}=4 e^{K} X^{I} \bar{X}^{J} h_{u v} k_{I}^{u} k_{J}^{v}-\frac{\imath}{s-\bar{s}} \eta^{I J} P_{I}^{x} P_{J}^{x}
$$

where the $P_{I}^{x}, x=1,2,3$ are the Killing prepotentials defined in appendix C.

For the present case where only the axionic shift (Peccei-Quinn) isometries $b^{A} \rightarrow$ $b^{A}+m_{I}^{A} \Lambda^{I}$ are gauged, the Killing prepotentials can be computed explicitly on the subspace $\mathcal{M}_{K 3}$ given in (3.3) which is spanned by the K3 moduli. The detailed calculation is presented in appendix $\mathbb{D}$ with the final result

$$
\eta^{I J} P_{I}^{x} P_{J}^{x}=\eta^{I J}\left(h_{u v} k_{I}^{u} k_{J}^{v}-m_{I}^{A} m_{J}^{B} \rho_{A B}\right)=\eta^{I J} h_{u v} k_{I}^{u} k_{J}^{v}
$$

where the last equation holds due to the constraint (3.24). Inserted into (3.32) one obtains

$$
\mathcal{V}_{\mathrm{E}}=\frac{\imath}{s-\bar{s}} h_{u v} k_{I}^{u} k_{J}^{v}\left[2 \frac{X^{I} \bar{X}^{J}+\bar{X}^{I} X^{J}}{X^{K} \bar{X}_{K}}-\eta^{I J}\right]=\frac{2}{(s-\bar{s})^{2}} h_{u v} k_{I}^{u} k_{J}^{v} I^{I J}
$$

where we used (3.5), 3.10) and (3.12). With the killing vectors which can be read off from (3.29) $k_{I}^{u}=m_{I}^{A}$, one immediately shows the equivalence of the potential derived from compactification (3.30) and the potential obtained from gauged supergravity (3.34).

Note that in the computation just described we had to set the moduli fields arising from the gauge bundle to zero. Only in this case we are able to compute the $P_{I}^{x}$. However, the consistency of the gauged supergravity with the compactification suggests that including the bundle moduli does not alter the $P_{I}^{x}$. It would be interesting to verify this explicitly.

\subsection{Fluxes simultaneously on $T^{2}$ and $\mathbf{K} 3$}

Let us now analyze the case where fluxes along both $T^{2}$ and $K 3$ are non-vanishing. We have seen in the previous two sections that the only candidates for turning on a background value are the field strengths of the vector fields. The consistency of the procedure was guaranteed by the Abelian gauge invariance which in turn was obtained by going to the Coulomb branch of the theory. On the other hand we have shown in section 2.2 that turning on fluxes in the torus compactifications generates the gauging of a non-Abelian group. Naively this would imply that no fluxes can be turned on in a further compactification on $K 3$. However, for the vector fields arising from the reduction of the antisymmetric tensor $B$ in ten dimensions non-trivial fluxes can be turned on as these vector fields appear in the action only via their Abelian field strength. This is a direct consequence of the two-form gauge invariance $B \rightarrow B+d \Lambda$ in $d=10$. Moreover if some of the fluxes in (3.15) vanish, the corresponding gauge fields also only appear via 
their Abelian field strength and hence it is possible to turn on fluxes along $K 3$ as in eq. (3.23) for all the gauge fields in $d=8$ which have an Abelian symmetry. The derivation of the effective action proceeds exactly as before with the result

$$
\begin{aligned}
S= & 2 \int \sqrt{-g} d^{4} x\left[\frac{1}{2} R+\frac{1}{8} I_{I J} F_{\mu \nu}^{I} F^{J \mu \nu}+\frac{1}{16} R_{I J} F_{\mu \nu}^{I} F_{\rho \lambda}^{J} \epsilon^{\mu \nu \rho \lambda}\right. \\
& \left.-g_{i j} \mathcal{D}_{\mu} z^{i} \mathcal{D}^{\mu} \bar{z}^{\bar{j}}-h_{u v} \mathcal{D}_{\mu} q^{u} \mathcal{D}^{\mu} q^{v}-\frac{\imath \mathcal{V}}{2(s-\bar{s})}\right]
\end{aligned}
$$

where the structure of the covariant derivatives is exactly as in (3.18) and (3.29) and the potential is the sum of the potentials given in eqs. (3.19), (3.30). Since also in the gauged supergravity the potential (C.12) is a sum of two terms the consistency with the reduction follows immediately from our previous analysis.

\subsection{Compactification on $K 3 \times S^{1}$}

Finally, let us briefly discuss the compactification of the heterotic string on $K 3 \times S^{1}$ to $d=5$. The derivation of the effective theory is very similar to the previous case and therefore we only highlight the differences. The five-dimensional gauged supergravity (with 8 supercharges) again contains a gravitational multiplet, $n_{V}$ vector multiplets and $n_{H}$ hypermultiplets. Now the scalars in the vector multiplets are real and their scalar manifold is a so called 'very special' manifold [36] while the hypermultiplets including their quaternionic geometry remain unchanged.

The effective theory of the the heterotic string compactified on $K 3 \times S^{1}$ without fluxes has been derived in ref. [37]. Including background fluxes again proceeds in two steps. First one compactifies the ten-dimensional heterotic string on an $S^{1}$ to $d=9$. In this step no fluxes can be turned on. Only by further compactification on $K 3$ all fluxes as in (3.23) can be chosen non-vanishing. Thus in the resulting five-dimensional theory the vector multiplet sector remains ungauged and only the scalar fields in the hypermultiplet become charged. This is in full agreement with the results of gauged five-dimensional supergravity as obtained in refs. [36, 38 40]. Furthermore, the covariant derivatives are exactly as in (3.29) and the scalar potential is found to be

$$
\mathcal{V}_{\mathrm{E}}=\frac{1}{s} h_{A B} m^{A I} m^{B J} M_{I J}
$$

where $M$ is the matrix (2.5) for the case where only one dimension is compactified on a circle. Its relation to the gauge couplings is

$$
M_{I J}=\frac{2}{s} I_{I J}, \quad s \equiv e^{-\frac{2}{3} \phi} .
$$

Note that we are using the basis where the antisymmetric tensor field $B_{\mu \nu}$ and not the dual gauge field appears explicitly in the action. The low energy effective action is described in terms of the derivatives of a cubic prepotential $V$ subject to the constraint $V=1$. For the heterotic string compactified on $K 3 \times S^{1}$ the scalars in the vector multiplets span the coset $S O\left(1, n_{V}\right) / S O\left(n_{V}\right)$ and the corresponding prepotential reads [37]

$$
V=S X^{I} X^{J} \eta_{I J}
$$


where the $X^{I}$ are now real. The gauge couplings are given by

$$
I_{I J}=\left.\partial_{I} \partial_{J} \ln V\right|_{V=1} \text {. }
$$

The consistency with the gauged supergravity of [36, 38 40] can be established using exactly same steps as in section 3.3 and so we will not present this in detail. We only note that the scalar potential of the $N=2, d=5$ gauged supergravity has a form similar to the one given in the four-dimensional case

$$
\mathcal{V}_{\mathrm{E}}=\left(2 I^{I J}-4 X^{I} X^{J}\right) P_{I}^{x} P_{J}^{x}+4 X^{I} X^{J} h_{u v} k_{I}^{u} k_{J}^{v}
$$

where $I^{I J}$ are the inverse gauge couplings. From (3.38) and (3.39) we learn

$$
I^{I J}=\frac{1}{s^{2}} \eta^{I K} \eta^{J L} I_{K L}=-\frac{1}{s} \eta^{I J}+2 X^{I} X^{J} .
$$

The geometry of the hypermultiplets is identical in $d=4,5,6$ and as a consequence the Killing vectors and also the computation of the Killing prepotentials $P_{I}^{x}$ presented in appendix D is unaltered. Using (D.13), (3.24) and (3.41) one then verifies the consistency of the potentials $(3.40)$ and $(3.36)$.

\section{Conclusions}

In this paper we studied the compactification of the heterotic string to $d=4,5$ with 8 or 16 preserved supercharges in the presence of background fluxes. We showed that in each case the bosonic sector of the low energy effective action is consistent with the corresponding gauged supergravity. However, the induced potential displays a dilaton instability and thus no Minkowski or anti-de Sitter ground state exists.

Interestingly, by turning on fluxes we were not able to recover all possible couplings of the gauged supergravities. In the case of 16 supercharges the gauged supergravity can have a constant term in the (string-frame) potential while in toroidal compactifications of the heterotic string this term is absent. Furthermore, in $d=5$ it is possible to gauge an Abelian group where the dual of the heterotic antisymmetric tensor field plays the role of the gauge field [24]. Also this type of gauging could not be obtained by turning on background fluxes.

In supergravities with 8 supercharges one can gauge the isometries of the vector- and hypermultiplet geometries. On the other hand, in $K 3$ compactifications of the heterotic string only a small subset of the isometries is gauged and in most cases these are axionic shift (Peccei-Quinn) symmetries.

For toroidal compactifications of the heterotic string in $d>5$ the bosonic action is also known but unfortunately the corresponding gauged supergravities have not been constructed yet and a hence a comparison cannot be performed. Finally, in compactifications of the heterotic string on $K 3$ to $d=6$ no background fluxes can be turned on at all since there are no Abelian gauge fields in $d=10$. 


\section{Appendix}

\section{A Conventions and notations}

Let us assemble our conventions in this appendix. The space time metric is taken to have the signature: $(-,+,+, \ldots+)$ while the Riemann tensor is defined to be $R_{\nu \lambda \rho}^{\mu}=$ $\partial_{\lambda} \Gamma_{\nu \rho}^{\mu}-\partial_{\nu} \Gamma_{\lambda \rho}^{\mu}+\Gamma_{\lambda \sigma}^{\mu} \Gamma_{\nu \rho}^{\sigma}-\Gamma_{\nu \sigma}^{\mu} \Gamma_{\lambda \rho}^{\sigma}$.

Throughout the paper we try to use the same indices for similar quantities but their range does change in the various compactifications. More specifically we use (if not specified otherwise)

$$
\begin{aligned}
\mu, \nu & =0, \ldots, d-1 \\
\alpha, \beta & =1, \ldots, n \\
a, b & =1, \ldots, 16 \\
I, J & =1, \ldots, 16+2 n \\
i, j & =1, \ldots, n_{V} \\
A, B & =1, \ldots, 22 \\
\Lambda, \Sigma & =1, \ldots, 4
\end{aligned}
$$

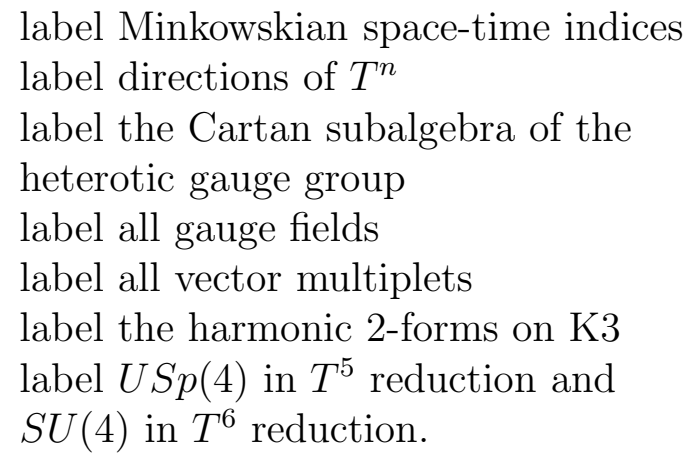

$g_{\mu \nu}$ denotes the space-time metric, while $G_{\alpha \beta}$ is used for the metric on the internal space.

\section{B $\quad N=4$ gauged supergravity in $d=5$}

The purpose of this appendix is to supply some of the details of the computation outlined in section 2.3. Let us first recall some basic facts about $U S p(4)$. It has an invariant symplectic two-form $\Omega$ which satisfies

$$
\Omega_{\Sigma \Delta} \Omega^{\Delta \Lambda}=-\Omega_{\Sigma}{ }^{\Delta} \Omega_{\Delta}{ }^{\Lambda}=-\delta_{\Sigma}{ }^{\Lambda}
$$

where the indices $\Sigma, \Lambda=1, \ldots, 4$ are raised and lowered with $\Omega$ and the convention for the matrix product is

$$
(A B)_{\Sigma}{ }^{\Lambda}=A_{\Sigma}{ }^{\Delta} B_{\Delta}{ }^{\Lambda}
$$

The other quantities we need in the main text are the five Euclidean $4 \times 4 \Gamma$-matrices satisfying

$$
\left\{\Gamma^{\alpha}, \Gamma^{\beta}\right\}_{\Sigma}{ }^{\Lambda}=2 \delta^{\alpha \beta} \delta_{\Sigma}{ }^{\Lambda}, \quad \operatorname{Tr}\left(\Gamma^{\alpha} \Gamma^{\beta}\right)=4 \delta^{\alpha \beta}, \quad \alpha=1, \ldots, 5 .
$$

Furthermore, the $\Gamma$-matrices are chosen to obey

$$
\left(\Gamma^{\alpha}\right)_{\Sigma \Lambda}=-\left(\Gamma^{\alpha}\right)_{\Lambda \Sigma} \quad \text { and } \quad\left(\Gamma^{\alpha}\right)_{\Sigma \Lambda} \Omega^{\Sigma \Lambda}=0 .
$$

\footnotetext{
${ }^{18}$ See appendix B of ref. [24].
} 
In order to relate the coordinates $L_{\Sigma \Lambda}^{I}$ to the matrix $M^{I J}$ used in [20] one defines the quantities $Z_{\alpha}^{I}$ by

$$
Z_{\alpha}^{I}=\frac{1}{2}\left(\Gamma^{\alpha}\right)_{\Lambda}{ }^{\Sigma} L_{\Sigma}^{I^{\Lambda}}{ }^{\Lambda}, \quad{L^{I}{ }_{\Sigma}{ }^{\Lambda}}^{\prime}=\frac{1}{2}\left(\Gamma^{\alpha}\right)_{\Sigma}{ }^{\Lambda} Z_{\alpha}^{I}, \quad I=1, \ldots, 26
$$

Inserted into (2.18) and using (B.4) one derives the constraint on the $Z_{\alpha}^{I}$ to be

$$
Z_{\alpha}^{I} Z_{\beta}^{J} \eta_{I J}=-\delta_{\alpha \beta}
$$

After eliminating $L_{i}{ }^{I}$ via the constraint given in (2.18) one typically encounters terms of the form $\operatorname{Tr}\left(L^{I_{1}} \ldots L^{I_{k}}\right)$ where the trace and the matrix multiplication is to be understood as acting on the indices $\Sigma, \Lambda$ according to (B.2). This can be easily computed using the trace properties of the $\Gamma$ matrices. As an example let us consider $k=2$. Using (B.3) one derives

$$
L_{I}{ }^{\Sigma \Lambda} L_{J \Sigma \Lambda}=\frac{1}{4} Z_{\alpha I} Z_{\beta J}\left(\Gamma^{\alpha}\right)^{\Sigma \Lambda}\left(\Gamma^{\beta}\right)_{\Sigma \Lambda}=Z_{\alpha I} Z_{\beta J} \delta^{\alpha \beta} \equiv Z_{I J}
$$

Finally, we dualize the spectator gauge field $a_{\mu}$ to an antisymmetric tensor $B_{\mu \nu}$. The terms in the Lagrangian (2.31) which contain the field $a$ are (written in form notation)

$$
\frac{1}{2} \int\left[-e^{\phi} G \wedge^{*} G+\eta_{I J} F^{I} \wedge F^{J} \wedge a\right]
$$

where $F^{I}=d A^{I}-\frac{1}{2} f_{J K}^{I} A^{J} \wedge A^{K}$. The last term in (B.8) can be rewritten in terms of the Chern-Simons term given in (2.12)

$$
\begin{aligned}
\eta_{I J} F^{I} \wedge F^{J} & =d \omega^{\mathrm{CS}} \\
\omega^{\mathrm{CS}} & =\eta_{I J} A^{I} \wedge F^{J}+\frac{1}{6} f_{I J K} A^{I} \wedge A^{J} \wedge A^{K} .
\end{aligned}
$$

Using (B.9) the equation of motion for a becomes

$$
d\left(e^{\phi *} G-\frac{1}{2} \omega_{c s}\right)=0 .
$$

We introduce a two-form $B$ and its three-form field strength $H$ such that

$$
d B=-e^{\phi *} G+\frac{1}{2} \omega_{c s}, \quad H=d B-\frac{1}{2} \omega_{c s} .
$$

The action for $H$ is now given by $\frac{1}{2} \int e^{-\phi} H \wedge^{*} H$ and we see that this is exactly the right kinetic term for $B$ as it is obtained from the compactification in eq. (2.9).

\section{C $\quad N=2$ gauged supergravity in $d=4$}

The purpose of this appendix is to give a short review of the $N=2$ supergravity in four dimensions [34, 41, 42]. A generic spectrum contains the gravitational multiplet, $n_{V}$ vector multiplets and $n_{H}$ hypermultiplets. The vector multiplets contain $n_{V}$ complex scalars 
$z^{i}, i=1, \ldots, n_{V}$ while the hypermultiplets contain $4 n_{H}$ real scalars $q^{u}, u=1, \ldots, 4 n_{H}$. Due to supersymmetry the scalar manifold factorizes

$$
\mathcal{M}=\mathcal{M}_{V} \otimes \mathcal{M}_{H}
$$

where the component $\mathcal{M}_{V}$ is a special Kähler manifold spanned by the scalars $z^{i}$ while $\mathcal{M}_{H}$ is a quaternionic manifold spanned by the scalars $q^{u}$.

A special Kähler manifold is a Kähler manifold whose geometry obeys an additional constraint [41]. This constraint states that the Kähler potential $K$ is not an arbitrary real function but determined in terms of a holomorphic prepotential $F$ according to

$$
K=-\ln \left(i \bar{X}^{I}\left(\bar{z}^{i}\right) F_{I}(X)-i X^{I}(z) \bar{F}_{I}(\bar{X})\right)
$$

The $X^{I}, I=0, \ldots, n_{V}$ are $\left(n_{V}+1\right)$ holomorphic functions of the $z^{i} . F_{I}$ abbreviates the derivative, i.e. $F_{I} \equiv \frac{\partial F(X)}{\partial X^{I}}$ and $F(X)$ is a homogeneous function of $X^{I}$ of degree 2, i.e. $X^{I} F_{I}=2 F$.

The $4 n_{H}$ scalars $q^{u}, u=1, \ldots, 4 n_{H}$ in the hypermultiplets are coordinates on a quaternionic manifold [42]. This implies the existence of three almost complex structures $\left(J^{x}\right)_{v}^{w}, x=1,2,3$ which satisfy the quaternionic algebra

$$
J^{x} J^{y}=-\delta^{x y}+i \epsilon^{x y z} J^{z}
$$

Associated with the complex structures is a triplet of Kähler forms

$$
K_{u v}^{x}=h_{u w}\left(J^{x}\right)_{v}^{w}
$$

where $h_{u w}$ is the quaternionic metric. The holonomy group of a quaternionic manifold is $S p(2) \times S p\left(2 n_{h}\right)$ and $K^{x}$ is identified with the field strength of the $S p(2) \sim S U(2)$ connection $\omega_{v}^{x}$, i.e.

$$
K^{x}=d \omega^{x}+\frac{1}{2} \epsilon^{x y z} \omega^{y} \wedge \omega^{z}
$$

The bosonic part of the $N=2$ action is given by

$$
S=\int d^{4} x \sqrt{-g}\left[\frac{1}{2} R+\frac{1}{4} I_{I J} F_{\mu \nu}^{I} F^{J \mu \nu}+\frac{1}{8} R_{I J} F_{\mu \nu}^{I} F_{\rho \lambda}^{J} \epsilon^{\mu \nu \rho \lambda}-g_{i \bar{\jmath}} \partial_{\mu} z^{i} \partial^{\mu} \bar{z}^{\bar{\jmath}}-h_{u v} \partial_{\mu} q^{u} \partial^{\mu} q^{v}\right]
$$

where $g_{i \bar{\jmath}}=\partial_{i} \partial_{\bar{\jmath}} K$ and the gauge coupling functions are given by

$$
\begin{aligned}
I_{I J} & \equiv \operatorname{Im} \mathcal{N}_{I J}, \quad R_{I J} \equiv \operatorname{Re} \mathcal{N}_{I J} \\
\mathcal{N}_{I J} & =\bar{F}_{I J}+2 i \frac{\operatorname{Im} F_{I K} \operatorname{Im} F_{J L} X^{K} X^{L}}{\operatorname{Im} F_{L K} X^{K} X^{L}}
\end{aligned}
$$

$F_{\mu \nu}^{0}$ denotes the field strength of the graviphoton.

One can gauge the isometries on the scalar manifold $\mathcal{M}$. Such isometries are generated by the Killing vectors $k_{I}^{u}(q), k_{I}^{i}(z)$

$$
\delta q^{u}=\Lambda^{I} k_{I}^{u}(q), \quad \delta z^{i}=\Lambda^{I} k_{I}^{i}(z)
$$


$k_{I}^{u}(q), k_{I}^{i}(z)$ satisfy the Killing equations which in $N=2$ supergravity can be solved in terms of four Killing prepotentials $\left(P_{I}, P_{I}^{x}\right)$. The Killing vectors on $\mathcal{M}_{V}$ are holomorphic and obey

$$
k_{I}^{i}(z)=g^{i \bar{j}} \partial_{\bar{j}} P_{I},
$$

while the Killing vectors on $\mathcal{M}_{H}$ are determined by a triplet of Killing prepotentials $P_{I}^{x}(q)$ via

$$
k_{I}^{u} K_{u v}^{x}=-D_{v} P_{I}^{x} \equiv-\left(\partial_{v} P_{I}^{x}+\epsilon^{x y z} \omega_{v}^{y} P_{I}^{z}\right) .
$$

Gauging the isometries (C.8) requires the replacement of ordinary derivatives by covariant derivatives in the action

$$
\partial_{\mu} q^{u} \rightarrow \mathcal{D}_{\mu} q^{u}=\partial_{\mu} q^{u}-k_{I}^{u} A_{\mu}^{I}, \quad \partial_{\mu} z^{i} \rightarrow \mathcal{D}_{\mu} z^{i}=\partial_{\mu} z^{i}-k_{I}^{i} A_{\mu}^{I}
$$

Furthermore the potential

$$
\begin{aligned}
\mathcal{V}_{\mathrm{E}} & =e^{K}\left[X^{I} \bar{X}^{J}\left(g_{\bar{\imath} j} k_{I}^{\bar{\imath}} k_{J}^{j}+4 h_{u v} k_{I}^{u} k_{J}^{v}\right)+g^{i \bar{\jmath}} D_{i} X^{I} D_{\bar{j}} \bar{X}^{J} P_{I}^{x} P_{J}^{x}-3 X^{I} \bar{X}^{J} P_{I}^{x} P_{J}^{x}\right] \\
& =e^{K} X^{I} \bar{X}^{J}\left(g_{\bar{\imath} j} k_{I}^{\bar{\imath}} k_{J}^{j}+4 h_{u v} k_{I}^{u} k_{J}^{v}\right)-\left(\frac{1}{2} I^{-1 I J}+4 e^{K} X^{I} \bar{X}^{J}\right) P_{I}^{x} P_{J}^{x},
\end{aligned}
$$

has to be added to the action in order to preserve supersymmetry. The bosonic part of the action of gauged $N=2$ supergravity is then given by

$$
\begin{aligned}
S= & 2 \int d^{4} x \sqrt{-g}\left[\frac{1}{2} R+\frac{1}{4} I_{I J} F_{\mu \nu}^{I} F^{J \mu \nu}+\frac{1}{8} R_{I J} F_{\mu \nu}^{I} F_{\rho \lambda}^{J} \epsilon^{\mu \nu \rho \lambda}\right. \\
& \left.-g_{i \bar{\jmath}} \mathcal{D}_{\mu} z^{i} \mathcal{D}^{\mu} \bar{z}^{\bar{\jmath}}-h_{u v} \mathcal{D}_{\mu} q^{u} \mathcal{D}^{\mu} q^{v}-\mathcal{V}_{\mathrm{E}}\right]
\end{aligned}
$$

\section{Computation of the Killing prepotential $P_{I}^{x}$}

In this appendix we compute explicitly the Killing prepotentials $P_{I}^{x}$ for the specific gauging which appears in section 3.3. To do this, one needs to solve the differential equation (C.10). Since we do not know the quaternionic manifold $\mathcal{M}_{H}$ for generic compactifications the $P_{I}^{x}$ cannot be easily determined. However, if we set to zero the moduli fields which arise from the gauge bundle, the hypermultiplet moduli space reduces to the coset $\mathcal{M}_{K 3}=S O(4,20) / S O(4) \times S O(20)$. In this case one can use the general formulas for homogenous spaces given in [34, 43]. More specifically for the $S U(2)$ connection $\omega^{x}$ one has

$$
\omega^{x}=-\frac{1}{2} \operatorname{Tr}\left(\theta \Sigma^{x}\right)
$$

where $\theta$ is a $4 \times 4$ matrix of one-forms which in terms of the coset representatives $Z$ reads

$$
\theta_{\alpha \beta}=Z_{\alpha I} \eta^{I J} d Z_{\beta J}, \quad \alpha, \beta=1, \ldots, 4
$$

The $\Sigma^{x}$ are the three self dual 't Hooft matrices as given in [34. Note that the quantities $Z$ are precisely the ones introduced in $(\mathrm{B} .5)$ for the coset $S O(5, \mathrm{~m}) / S O(5) \times S O(\mathrm{~m})$. However, from the compactification on $K 3$ one does not obtain directly the coset representatives $Z$, but a $S O(4,20)$ matrix $\mathcal{M}^{-1}$ which should satisfy a condition similar to (2.25). From the discussions in section 3.3 it is clear that the scalars coming from the 
reduction of the antisymmetric two-form $B$ play a crucial role in the gauged theory as they are the only scalars which become charged when fluxes are turned on along $K 3$. That is why we are going to choose the following parameterization of the matrix $\mathcal{M}^{-1}$

$$
\mathcal{M}^{-1}=\left(\begin{array}{ccc}
v^{-1} & -v^{-1} b^{T} \eta & -\frac{1}{2} v^{-1}\left(b^{T} \eta b\right) \\
-v^{-1} \eta b & v^{-1} \eta b b^{T} \eta+N & \frac{1}{2} v^{-1}\left(b^{T} \eta b\right) \eta b+N b \\
-\frac{1}{2} v^{-1}\left(b^{T} \eta b\right) & \frac{1}{2} v^{-1}\left(b^{T} \eta b\right) b^{T} \eta+b^{T} N & \frac{1}{4} v^{-1}\left(b^{T} \eta b\right)^{2}+b^{T} N b+v
\end{array}\right)
$$

which precisely singles out the $b$ 's while the dependence on the other 57 moduli of $K 3$ is only via the $S O(3,19)$ matrix $N$ defined in (3.31). ( $v$ is the volume of the $K 3$.) The matrices $\mathcal{M}$ and $N$ are elements of $S O(4,20)$ and $S O(3,19)$ respectively in that they obey

$$
\mathcal{M}^{-1} \mathcal{L} \mathcal{M}^{-1 T}=\mathcal{L}, \quad N \rho N^{T}=\rho .
$$

$\mathcal{L}$ and $\rho$ are $S O(4,20)$ respectively $S O(3,19)$ metrics given by

$$
\mathcal{L}=\left(\begin{array}{ccc}
0 & 0 & 1 \\
0 & \rho & 0 \\
1 & 0 & 0
\end{array}\right), \quad \rho_{A B}=\int_{K 3} \Omega_{A} \wedge \Omega_{B} .
$$

Let us define the $4 \times 24$ matrix

$$
Z=\left(\begin{array}{ccc}
\frac{1}{\sqrt{2}} v^{-\frac{1}{2}} & -\frac{1}{\sqrt{2}} v^{-\frac{1}{2}} b^{T} \eta & -\frac{1}{2 \sqrt{2}} v^{-\frac{1}{2}}\left(b^{T} \eta b\right)-\frac{1}{\sqrt{2}} v^{\frac{1}{2}} \\
0 & u & u b
\end{array}\right),
$$

where $u$ is a $3 \times 22$ matrix satisfying the analog of 2.25$)$

$$
2 u^{T} u=N-\rho .
$$

Furthermore $u$ depends only on the $57 K 3$ moduli but not on $b$. It is easy to check that the matrix $Z$ defined in this way satisfies

$$
2 Z_{\alpha I} Z_{\beta J} \delta^{\alpha \beta}=\mathcal{M}_{I J}^{-1}-\mathcal{L}_{I J}
$$

Inserting the coset representative $Z$ given in (D.6) into (D.2) the $4 \times 4$ matrix $\theta$ is found to be

$$
\theta=\left(\begin{array}{cc}
0 & \frac{1}{\sqrt{2}} v^{-\frac{1}{2}} d b^{T} u \\
-\frac{1}{\sqrt{2}} v^{-\frac{1}{2}} u d b & u \rho d u^{T}
\end{array}\right) .
$$

In order to determine the $P_{I}^{x}$ via eq. (C.10) we only need the $S U(2)$ connection $\omega^{x}$ in the direction of the 22 axionic fields $b^{A}$. Inserting (D.9) into (D.1) one finds

$$
\omega_{A}^{1}=\frac{u_{A}^{1}}{\sqrt{2 v}}, \quad \omega_{A}^{2}=-\frac{u_{A}^{2}}{\sqrt{2 v}}, \quad \omega_{A}^{3}=\frac{u_{A}^{3}}{\sqrt{2 v}} .
$$

The solution of (C.10) is possible even if an explicit form of the matrix $u$ is not given. This is due to the fact that $\omega_{a}^{x}$ does not depend on $b$ and that the Killing vectors point only in the $b$ direction. Using (C.5), $k_{I}^{u}=m_{I}^{A}$ and $\partial_{A} \omega_{v}^{x}=0$ we find

$$
k_{I}^{u} K_{u v}^{x}=m_{I}^{A}\left(\partial_{A} \omega_{v}^{x}-\partial_{v} \omega_{A}^{x}+\epsilon^{x y z} \omega_{A}^{y} \omega_{v}^{z}\right)=-D_{v}\left(m_{I}^{A} \omega_{A}^{x}\right)=-D_{v}\left(k_{I}^{u} \omega_{u}^{x}\right),
$$


which in turn implies that $(\overline{C .10})$ is solved by

$$
P_{I}^{x}=k_{I}^{u} \omega_{u}^{x}
$$

The term $P_{I}^{x} P_{J}^{x}$ which appears in the $N=2$ potential C.12 now yields

$$
P_{I}^{x} P_{J}^{x}=k_{I}^{u} k_{J}^{v} \omega_{u}^{x} \omega_{v}^{x}=\frac{1}{2} v^{-1} u_{A}^{x} u_{B}^{x} m_{I}^{A} m_{J}^{B}=\frac{1}{4} v^{-1}\left(N_{A B}-\rho_{A B}\right) m_{I}^{A} m_{J}^{B},
$$

where we used (D.7) and (D.10). Inserting (3.31) we finally obtain

$$
P_{I}^{x} P_{J}^{x}=\left(h_{A B}-\frac{\rho_{A B}}{4 v}\right) m_{I}^{A} m_{J}^{B}=h_{u v} k_{I}^{u} k_{J}^{v}-\frac{\rho_{A B} m_{I}^{A} m_{J}^{B}}{4 v} .
$$

Using (3.24) this further simplifies and we have

$$
\eta^{I J} P_{I}^{x} P_{J}^{x}=h_{u v} k_{I}^{u} k_{J}^{v} \eta^{I J}
$$

\section{Acknowledgments}

This work is supported by the DFG (German Science Foundation), GIF (GermanIsraeli Foundation for Scientific Research), the European RTN Program HPRN-CT-200000148 and the DAAD (German Academic Exchange Service).

We thank C. Bachas, E. Bergshoeff, B. Gunara, M. Haack, C. Herrmann, A. Klemm, D. Lüst, T. Mohaupt, H. Singh, S. Theisen and M. Zagermann for useful conversations.

\section{References}

[1] D. J. Gross, J. A. Harvey, E. J. Martinec, and R. Rohm, "Heterotic string theory 1. The free heterotic string," Nucl. Phys. B256 (1985) 253.

[2] P. Candelas, G. T. Horowitz, A. Strominger, and E. Witten, "Vacuum configurations for superstrings," Nucl. Phys. B258 (1985) 46.

[3] R. Rohm and E. Witten, "The antisymmetric tensor field in superstring theory," Ann. Phys. 170 (1986) 454.

[4] A. Strominger, "Superstrings with torsion," Nucl. Phys. B274 (1986) 253.

[5] B. de Wit, D. J. Smit, and N. D. Hari Dass, "Residual supersymmetry of compactified d = 10 supergravity," Nucl. Phys. B283 (1987) 165.

[6] J. Polchinski and A. Strominger, "New vacua for type II string theory," Phys. Lett. B388 (1996) 736, hep-th/9510227.

[7] C. Bachas, "A way to break supersymmetry," hep-th/9503030.

[8] J. Michelson, "Compactifications of type IIB strings to four dimensions with nontrivial classical potential," Nucl. Phys. B495 (1997) 127, hep-th/9610151. 
[9] I. Antoniadis, E. Gava, K. S. Narain, and T. R. Taylor, "Duality in superstring compactifications with magnetic field backgrounds," Nucl. Phys. B511 (1998) 611, hep-th/9708075.

[10] T. R. Taylor and C. Vafa, "RR flux on Calabi-Yau and partial supersymmetry breaking," Phys. Lett. B474 (2000) 130, hep-th/9912152.

[11] P. Mayr, "On supersymmetry breaking in string theory and its realization in brane worlds," Nucl. Phys. B593 (2001) 99, hep-th/0003198.

[12] G. Curio, A. Klemm, D. Lüst, and S. Theisen, "On the vacuum structure of type II string compactifications on Calabi-Yau spaces with H-fluxes," Nucl. Phys. B609 (2001) 3, hep-th/0012213.

[13] S. B. Giddings, S. Kachru, and J. Polchinski, "Hierarchies from fluxes in string compactifications," hep-th/0105097.

[14] J. Scherk and J. H. Schwarz, "How to get masses from extra dimensions," Nucl. Phys. B153 (1979) 61.

[15] E. Bergshoeff, M. de Roo, M. B. Green, G. Papadopoulos, and P. K. Townsend, "Duality of type II 7-branes and 8-branes," Nucl. Phys. B470 (1996) 113, hep-th/9601150.

[16] I. V. Lavrinenko, H. Lu and C. N. Pope, "From topology to generalised dimensional reduction," Nucl. Phys. B492 (1997) 278, hep-th/9611134.

[17] C. M. Hull and P. K. Townsend, "Unity of superstring dualities," Nucl. Phys. B 438 (1995) 109, hep-th/9410167;

E. Witten, "String theory dynamics in various dimensions," Nucl. Phys. B443 (1995) 85, hep-th/9503124.

[18] For a review see, for example, B. de Wit and J. Louis, "Supersymmetry and dualities in various dimensions," hep-th/9801132 and references therein.

[19] E. Bergshoeff, M. de Roo, and E. Eyras, "Gauged supergravity from dimensional reduction," Phys. Lett. B413 (1997) 70, hep-th/9707130.

[20] N. Kaloper and R. C. Myers, "The $\mathrm{O}(\mathrm{dd})$ story of massive supergravity," JHEP 05 (1999) 010, hep-th/9901045.

[21] M. Haack, J. Louis, and H. Singh, "Massive type IIA theory on K3," JHEP 04 (2001) 040, hep-th/0102110.

[22] E. Kiritsis and C. Kounnas, "Perturbative and non-perturbative partial supersymmetry breaking: $\mathrm{N}=4 \rightarrow \mathrm{N}=2 \rightarrow \mathrm{N}=1$," Nucl. Phys. B503 (1997) 117, hep-th/9703059.

[23] G. Curio, A. Klemm, B. Körs, and D. Lüst, "Fluxes in heterotic and type II string compactifications," hep-th/0106155.

[24] G. Dall'Agata, C. Herrmann, and M. Zagermann, "General matter coupled N = 4 gauged supergravity in five dimensions," hep-th/0103106. 
[25] L. Andrianopoli, R. D'Auria, and S. Vaula, "Matter coupled F(4) gauged supergravity lagrangian," JHEP 05 (2001) 065, hep-th/0104155.

[26] K. S. Narain, "New heterotic string theories in uncompactified dimensions < 10," Phys. Lett. B169 (1986) 41;

K. S. Narain, M. H. Sarmadi, and E. Witten, "A note on toroidal compactification of heterotic string theory," Nucl. Phys. B279 (1987) 369.

[27] J. Maharana and J. H. Schwarz, "Noncompact symmetries in string theory," Nucl. Phys. B390 (1993) 3, hep-th/9207016.

[28] M. de Roo, "Gauged N=4 matter couplings," Phys. Lett. B156 (1985) 331;

M. de Roo, "Matter coupling in N=4 supergravity," Nucl. Phys. B255 (1985) 515;

E. Bergshoeff, I. G. Koh, and E. Sezgin, "Coupling of Yang-Mills to $\mathrm{N}=4$, d = 4 supergravity," Phys. Lett. B155 (1985) 71.

[29] P. Wagemans, "Breaking of $\mathrm{N}=4$ supergravity to $\mathrm{N}=1, \mathrm{~N}=2$ at $\Lambda=0$," Phys. Lett. B206 (1988) 241.

[30] A. Ceresole, R. D'Auria, S. Ferrara and A. Van Proeyen, "Duality transformations in supersymmetric Yang-Mills theories coupled to supergravity," Nucl. Phys. B444 (1995) 92, hep-th/9502072.

[31] B. de Wit, V. Kaplunovsky, J. Louis, and D. Lüst, "Perturbative couplings of vector multiplets in $\mathrm{N}=2$ heterotic string vacua," Nucl. Phys. B451 (1995) 53, hep-th/9504006.

[32] I. Antoniadis, S. Ferrara, E. Gava, K. S. Narain and T. R. Taylor, "Perturbative prepotential and monodromies in $\mathrm{N}=2$ heterotic superstring," Nucl. Phys. B447 (1995) 35, hep-th/9504034.

[33] For a review see, for example, J. Louis and K. Foerger, "Holomorphic couplings in string theory," Nucl. Phys. Proc. Suppl. 55B (1997) 33, hep-th/9611184;

D. Lüst, "String vacua with $\mathrm{N}=2$ supersymmetry in four dimensions," hep-th/9803072, and references therein.

[34] For a review see, for example, L. Andrianopoli, M. Bertolini, A. Ceresole, R. D'Auria, S. Ferrara, P. Fre, and T. Magri, " $\mathrm{N}=2$ supergravity and $\mathrm{N}=2$ super YangMills theory on general scalar manifolds: Symplectic covariance, gaugings and the momentum map," J. Geom. Phys. 23 (1997) 111, hep-th/9605032, and references therein.

[35] N. Seiberg, "Observations On The Moduli Space Of Superconformal Field Theories," Nucl. Phys. B303 (1988) 286.

[36] M. Gunaydin, G. Sierra, and P. K. Townsend, "Gauging the d = 5 Maxwell- Einstein supergravity theories: More on Jordan algebras," Nucl. Phys. B253 (1985) 573.

[37] I. Antoniadis, S. Ferrara, and T. R. Taylor, "N=2 heterotic superstring and its dual theory in five dimensions," Nucl. Phys. B460 (1996) 489, hep-th/9511108. 
[38] A. Lukas, B. A. Ovrut, K. S. Stelle, and D. Waldram, "Heterotic M-theory in five dimensions," Nucl. Phys. B552 (1999) 246, hep-th/9806051.

[39] M. Gunaydin and M. Zagermann, "The gauging of five-dimensional, $\mathrm{N}=2$ MaxwellEinstein supergravity theories coupled to tensor multiplets," Nucl. Phys. B572 (2000) 131, hep-th/9912027;

M. Gunaydin and M. Zagermann, "The vacua of $5 \mathrm{~d}, \mathrm{~N}=2$ gauged YangMills/Einstein/tensor supergravity: Abelian case," Phys. Rev. D62 (2000) 044028, hep-th/0002228.

[40] A. Ceresole and G. Dall'Agata, "General matter coupled $\mathrm{N}=2, \mathrm{D}=5$ gauged supergravity," Nucl. Phys. B585 (2000) 143, hep-th/0004111.

[41] B. de Wit and A. Van Proeyen, "Potentials and symmetries of general gauged N=2 supergravity - Yang-Mills models," Nucl. Phys. B245 (1984) 89;

L. Castellani, R. D'Auria, and S. Ferrara, "Special Kähler geometry: An intrinsic formulation from N=2 space-time supersymmetry," Phys. Lett. B241 (1990) 57;

L. Castellani, R. D'Auria, and S. Ferrara, "Special geometry without special coordinates," Class. Quant. Grav. 7 (1990) 1767.

[42] J. Bagger and E. Witten, "Matter Couplings In N=2 Supergravity ," Nucl. Phys. B222 (1983) 1;

B. de Wit, P. G. Lauwers, and A. Van Proeyen, "Lagrangians Of N=2 Supergravity - Matter Systems," Nucl. Phys. B255 (1985) 569;

R. D'Auria, S. Ferrara and, P. Fre, "Special and quaternionic isometries: General couplings in $\mathrm{N}=2$ supergravity and the scalar potential," Nucl. Phys. B359 (1991) 705 .

[43] P. Fre, L. Girardello, I. Pesando, and M. Trigiante, "Partial N=2 $\rightarrow \mathrm{N}=1$ local supersymmetry breaking and solvable Lie algebras," hep-th/9611188. 